\title{
Research on New Reinforcement for Basic Foundation of Existing Buildings
}

\author{
Xingdong $\mathrm{Ma}^{1, \mathrm{a}}$, Yu Zhang ${ }^{2, \mathrm{~b}}$, \\ ${ }^{1}$ No. 1 Huanhua North Road of Chengdu, China \\ ${ }^{2}$ No. 55 North Road of Chengdu, China \\ a81257768@qq.com, b273652902@qq.com
}

Keywords: existing building; new construction; foundations; reinforcement; steel pipe pile

Abstract. It has become a novel and universal engineering problem that the new construction caused uneven foundation settlement of existing building foundations. This paper relies on the actual project that the tilt of existing buildings caused by a new tunnel. For the uneven settlement of existing building foundation, the reinforcement treatment is carried out. A new rapid reinforcement method of using steel pipe pile with H-beam is proposed, and the reinforcement mechanism of the method is clarified. And the reinforcing effects of the method are verified by field static load test and site monitoring data. The settlement laws of existing buildings in three stages, which are before reinforcement, reinforcement period, after reinforcement, are revealed. The rationality of the reinforcement method is fully demonstrated. This paper provides a fast, rational and effective reference method for solving similar engineering problems in the future.

\section{Introduction}

In recent years, with the rapid development of China's urban construction, due to the new buildings, subway and other projects need for excavation in the vicinity of existing buildings, which caused uneven settlement of existing buildings and lead to cracking accident of its original structure, has gradually become a new and common engineering problems.

Currently, researches ${ }^{[1-7]}$ on foundation reinforcement method for existing buildings, have achieved many results. For example: A new composite grouting was presented by Han etc. ${ }^{[1]}$, that Chronologically combined high pressure jet grouting and the static pressure grouting to form a new grouting method, he focuses on the mechanism and characteristics of grouting compound, and use it to solve engineering problems; Wu etc. ${ }^{[2]}$ used the single hole composite bolt pile to reinforcing foundation, and studied on the reinforcement mechanism of the single hole composite bolt pile; For different practical engineering, $\mathrm{Xu}$ and Zhang all carried out application of the anchor static pressure piles for foundation reinforcement of existing building respectively, and it provides a good research ideas for the actual project without piling conditions; $\mathrm{Nie}^{[5]}$ conducted research on reinforcement methods of buildings foundations within its construction, and discussed in detail the common building foundation reinforcement construction methods, such as grouting and static pressure piles. Overall, the common reinforcement method of foundation can be divided into: grouting method, grouting foundation reinforcement method, anchor static pressure pile method, static pressure pile pit method, increase the foundation area method, deepen basic law, roof Pile Method and lime pile method. The above reinforcement methods researches are mostly carried out for the reasons of uneven foundation settlement or unreasonable infrastructure. And on the engineering problem of new construction led to the foundations of existing building need for reinforcement, the study is relatively small. 
Therefore, in order to solve such new engineering problems, the research on new foundation reinforcement method should be carried out urgently. This paper relies on an actual project which a new tunnel causes the adjacent existing buildings to tilt in Suining, Sichuan. The reinforcement method research for uneven settlement of foundation is carried out. A new rapid reinforcement method of using steel pipe pile with H-beam to connect to the original foundation, is proposed based on the actual project. And the actual reinforcement effects of the method are verified by the site load test and monitoring data. This method provides a fast, rational and effective research idea for the design and construction of emergency reinforcement project of foundations.

\section{Project overview}

This paper relies on an actual project which a new tunnel leads to tilt and structural cracks of the adjacent existing building in Suining, Sichuan. The edges of the new tunnel and the existing building are 4 meters apart, as shown in Fig. 1. The underlying structure of existing buildings is reinforced concrete frame structure, while the upper structure of the building is the $6 \sim 7$ layer (local) masonry structure, and its base form are reinforced concrete independent foundation and strip foundation under column and wall. SMW continuous wall construction has been completed before the tunnel excavation. When the tunnel is under construction, there are different degrees of cracks in the main structure of the existing building, such as the floor, the ground beam, the wall, the roof and so on. And this has caused a serious security problem for existing buildings.



Based on the existing survey and design information, the rock stratum of existing building area is mainly composed of artificial fill, silty clay, slightly dense pebbles, medium dense pebble. And the physical and mechanical parameters of the rock stratum are shown in Table 1.

Table 1 Statistical table of physical and mechanical index of rock and soil

\begin{tabular}{ccccc}
\hline Stratum & $\gamma\left(\mathrm{kN} / \mathrm{m}^{3}\right)$ & $\mathrm{c} / \mathrm{kPa}$ & $\varphi /{ }^{\circ}$ & $\mathrm{fa} / \mathrm{kPa}$ \\
\hline Artificial fill & 18 & 5 & 18 & - \\
Silty clay & 19.1 & 26 & 16 & 130 \\
Slightly dense pebbles & 21 & 0 & 35 & 300 \\
Medium dense pebble & 22 & 0 & 40 & 550 \\
\hline
\end{tabular}

The $\mathrm{J} 1$ to $\mathrm{J} 8$ are a monitoring points in Fig. 1, and the site monitoring shows that, the side of existing building near the new construction project appears to sink, the settlement amount is $22.8 \mathrm{~mm}$ to $29.7 \mathrm{~mm}$, and the side deviating from the new project appears to lift, the total amount of the lift is $0.7 \mathrm{~mm}$ to $5.9 \mathrm{~mm}$. Therefore, the existing building appears uneven settlement. The identification analysis shows that, the construction of the new project results in the expansion of the part of the original building cracks, and causes it to appear apparent overall tilt. This has a serious 
security risk to the use of its main body structure, so that the reinforcement for existing building foundation is urgent.

\section{Reinforcement scheme study}

Combined with the distribution of neighboring buildings and the rock strata of the area, it finds that conventional reinforcement methods can not meet the requirements of the rapid and effective reinforcement of the project. While considering that the building is generally based on the sand and gravel as bearing layer in Chengdu area, and based on the researches ${ }^{[8-9]}$ of the existing methods of steel pipe pile reinforcement, this paper will carry out research on new reinforcement method with the use of steel pipe pile, for the practical engineering problems.

The basic form of the existing building is mainly based on strip foundation. The ring beam is above on foundation, and the frame column or wall are upon on the ring beams. The reinforcement method (shown in Fig.2 and Fig.3) is as follows: First of all, holes are drilled on the strip foundation, and the end of the steel pipe pile with diameter of $140 \mathrm{~mm}$ and wall thickness of $8 \mathrm{~mm}$ is entered into medium dense pebble by piling; Secondly, the H-beam is welded at the top of the pile, and which is parallel to the strip foundation; Then, the $20 \mathrm{~mm}$ third grade bars are connected to the H-beam, meanwhile which are implanted into the strip foundation, and ensure that the anchoring depth is not less than 22 times the diameter of the steel bar; Finally, the whole pile top and the H-beam are enclosed by $\mathrm{C} 20$ concrete.

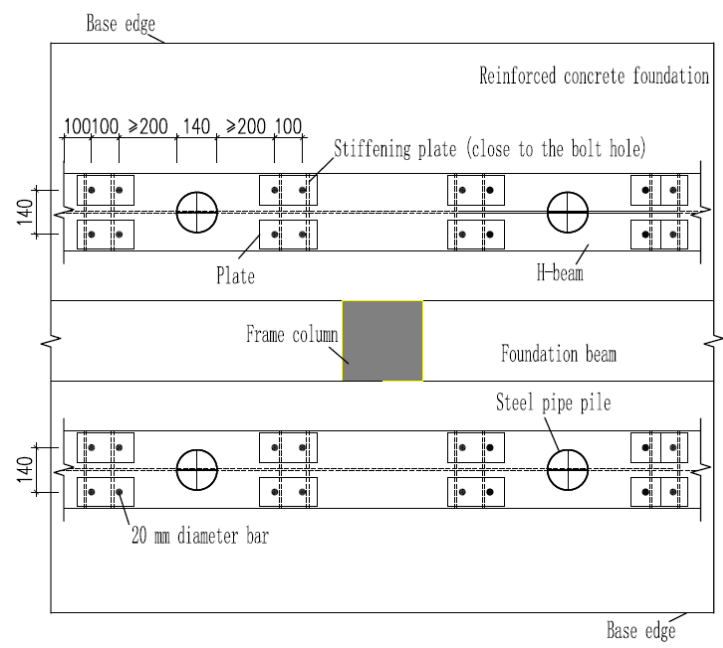

Fig. 2 The plan of foundations reinforcement of using steel pipe pile with H-beam

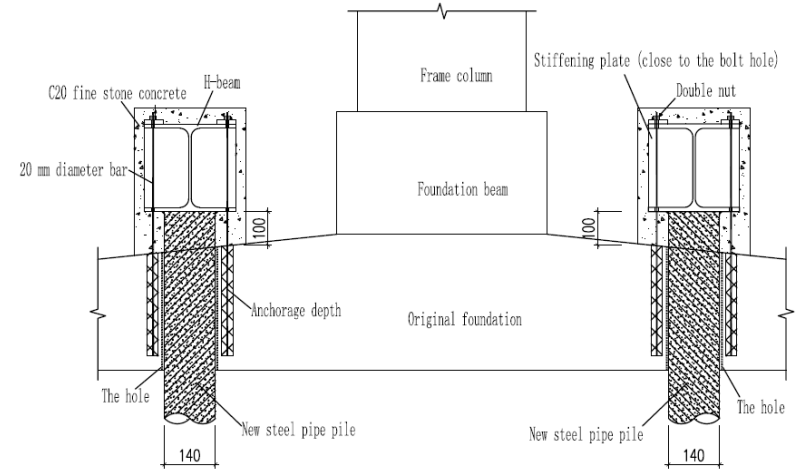

Fig. 3 The elevation of foundations reinforcement of using steel pipe pile with H-beam

Firtly, the steel pipe piles adopted in this method can be quickly entered into the bearing stratum by hammering. And all the pipe pile tops are connected by H-beams along the strip foundation. Then, the H-beams are connected with the strip foundation by bolt connection and 
implant anchorage. Finally, the steel pipe piles and strip foundation form an overall force structure, and share the upper structure load together. The method proposed in this paper is easy to operate, and can quickly coordinate the overall stiffness of the foundation, so as to reduce the uneven settlement of buildings in order to achieve the reinforcement effect.

\section{Verification analysis of reinforcement effect}

The new reinforcement method previously introduced which is composed by steel pipe piles and H-beams, has been applied to actual rescue engineering. For further detect and evaluate the effect of reinforcement, the confirmatory analysis is conducted by the combination of load test and field monitoring.

\section{Load test detection}

After the steel pipe piles entering into the bearing stratum, according to the "Technical code for building pile foundations " ${ }^{[10]}$, this paper is based on the test requirements of $1 \%$ and no less than 3 piles, the vertical static load tests of single pile are carried out, as shown in Fig.4.
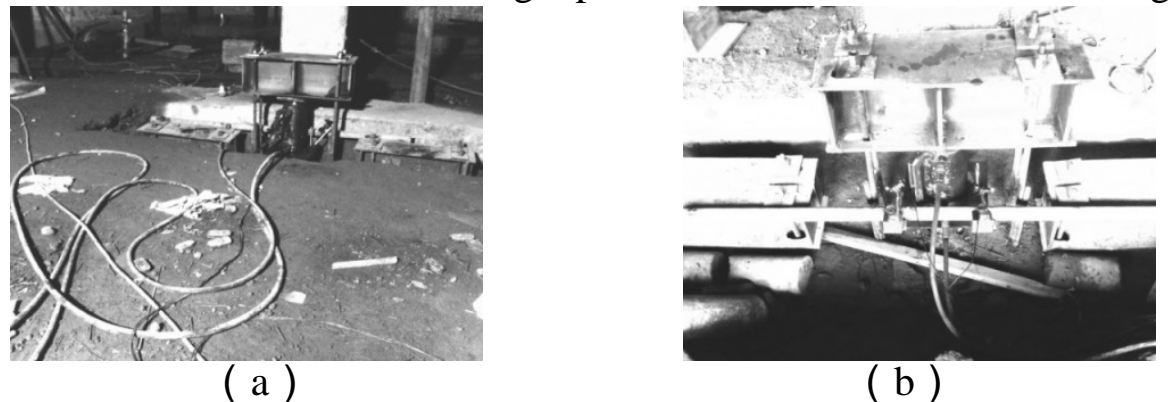

Fig. 4 Load test detection for steel pipe pile

According to the steel pipe pile load tests, it can be known that, after the steel pipe piles getting into the medium dense pebble, the characteristic value of vertical bearing capacity of single pile reaches $180 \mathrm{kN}$, which meets the requirements of foundation bearing capacity.

Field monitoring and evaluation

For the reinforcement method, the continuous building settlement deformation observation is carried out in the entire foundation reinforcement process (5-30 to 6-21) and after the reinforcement. The monitoring point position are shown in Fig. 1 (J1 to J8), and the monitoring results are shown in Fig. 5 (subsidence is negative, uplift is positive).

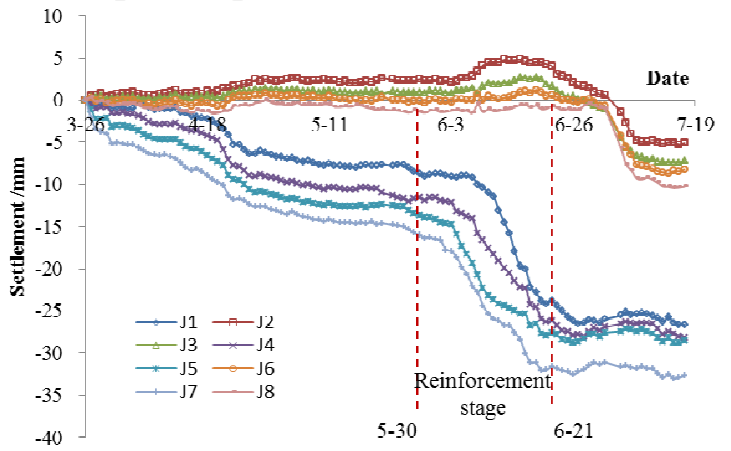

Fig. 5 Settlement observation of existing building

It can be seen from Fig. 5 that, since after the excavation (3-26) of the new tunnel, the monitoring points (such as J1,J4、J5, J7) near the tunnel excavation line show settlement phenomenon. Among the monitoring points, the settlement rate of $\mathrm{J} 7$ point (at the upper right corner of housing plane) is the fastest and the settlement is the largest. Before the reinforcement starts 
(5-30), the largest settlement reaches $16.3 \mathrm{~mm}$; The monitoring points (such as J2、J3、J6、J8) deviating from the tunnel line show uplift phenomenon. The uplift rate of J7 point at lower left corner of housing plane is the fastest and uplift is the largest, and the maximum uplift amount is $2.4 \mathrm{~mm}$ before reinforcement. It can be seen that the existing building appears obvious uneven settlement after the excavation of tunnel, and which tilts toward the tunnel foundation pit.

In Fig. 5, 5-30 to 6-21 is the reinforcing period of existing building. Sedimentation rate and settlement of settlement points above increased significantly at the reinforcing period, and the uplift phenomenon also has a certain development. This is mainly due to the pipe piles in the process of being hammered into the pile bearing stratum, which has produce a certain amount of disturbance to the existing building foundation, and which bring additional uneven settlement; However, after the reinforcement method of steel pipe piles with H-beam was adopted, the settlement and uplift phenomenon of existing building both weakened rapidly. Subsequently the original uplift parts show a certain settlement, and eventually remain stable. The entire consolidation period is only 22 days.

It can be seen that, the new reinforcement method proposed in this paper, can quickly and effectively deal with the engineering disease problems that new project leads to uneven settlement of existing buildings. And it provides a good reference for solving the similar problems in the future.

\section{Summary}

This paper relies on a practical project which a new tunnel causes the adjacent buildings to tilt. The reinforcement method research for uneven settlement of foundation is carried out. A new rapid reinforcement method of using steel pipe pile with H-beam is proposed. The reinforcement mechanism of the method has been fully clarified. The method has been verified by the field static load test and the monitoring data. The rapidity and effectiveness of this method have been verified by field static load test and monitoring data. The settlement laws of existing buildings in three stages, which are before reinforcement, reinforcement period, after reinforcement, are revealed. And the rationality of the reinforcement method is fully demonstrated. The new reinforcement method proposed in this paper, can provides a fast, rational and effective reference method for solving similar engineering problems in the future.

\section{References}

[1] HAN Jin-tian, LIU Hong-bo. Application of composite grouting method in foundation reinforcement[J]. Geological Exploration For Non-ferrous Metals, 2001, 4(9):42-45.

[2] WU Shun-chuan, JIANG Chun-lin, ZHANG You-pa. Application of composite anchor pile in foundation reinforcement[J]. Journal of University of Science and Technology Beijing, 2003, 25(5):398-401.

[3] XU Xing-hua, FU Zhao-ming, WU Jin-zhan. Application of anchor static pressure pile in building foundation reinforcement[J]. Building Structure, 2004, 34(12):22-23.

[4] ZHANG Na. Application of anchor static pressure pile method in foundation reinforcement of existing building foundation[J]. Science and Technology of West China, 2011, (7):23-24.

[5] Nie Guo-dong. Research on technology and strengthening technology of foundation construction technology[J]. Science and technology \& Enterprises, 2013, (17):177-179. 
[6] ChenAi-xin, LiuChang-qing. Geotechnical investigation for strengthening and remedy of the existing building foundation and scheme options for foundation reinforcement[J]. Geotechnical Investigation \& Surveying, 2010, (04):27-30.

[7] JGJ 123-2012, Technical Code for Improvement of Soil and Foundation of Existing Buildings (S).

[8] TANG Chuan-zheng, SHU Wu-tang. APPLICATION OF MICRO STEEL PIPE PILES TO DEAL WITH PIT DESTRUCTION[J]. Chinese Journal of Rock Mechanics and Engineering, 2005, 24, (S2): 5459-5463.

[9] YAN Ming. Test Research On Reinforcement Sunk Foundation With Pre-Stressed Micro Steel Pipe Piles[J]. Architecture Technology, 2008, 39, (6): 465-466.

[10]JGJ 94-2008, Technic al code for building pile foundations (S). 